\title{
Idiopathic fibrosing hypertrophic gastritis: a new entity that mimics linitis plastica carcinoma
}

The author reports a case and proposes a hitherto undescribed entity of "idiopathic fibrosing hypertrophic gastritis". This entity is characterized by diffuse severe submucosal fibrosis and hypertrophy of the muscularis propria of the stomach, of unknown etiology. This gastritis characteristically simulates linitis plastica carcinoma and causes pyloric stenosis, endoscopically and grossly.

A 55-year-old man presented with loss of appetite. His past history and blood data suggested no relevant factors. Endoscopy showed severe pyloric stenosis and strongly suggested linitis plastica carcinoma ( Fig. 1a); however, biopsies were negative. Total gastrectomy was performed. Pathologic gross examination showed marked thickening of the entire gastric wall ( Fig. 1b). Microscopic examination of the whole stomach showed diffuse marked submucosal fibrosis with inflammation ( $\bullet$ Fig. 1 c) and diffuse severe hypertrophy of the muscularis propria ( Fig. 1d). The mucosa was free of ulcers and ulcer scars. No carcinoma cells were recognized on microscopic and immunohistochemical studies (cytokeratins, p53, MIB-1, and CEA). Special and immunohistochemical stains showed no amyloid or organisms (Helicobacter pylori, Spirochaeta spp., Gram-positive bacteria, acid-fast bacteria, cytomegalovirus, and fungi), so the etiology was unknown. Cases like the present one are extremely rare. Such cases of gastritis simulating linitis plastica carcinoma have been reported only twice, in corrosive gastritis [1] and in cytomegalovirus gastritis [2]. The etiology of the present case is unknown, and only speculative. Congenital pyloric stenosis is unlikely because of the patient's age. Healed gastric ulcer is unlikely because the patient's past history revealed no relevant factors, and also because it is unlikely that gastric ulcer would lead to severe fibrosis and muscular hypertrophy in the entire stomach. The author speculates that repeated unknown attacking agents caused fibrosis of the gastric walls, causing submucosal fibrosis and pyloric stenosis. To overcome the stenosis, the muscular layer later became hypertrophic.
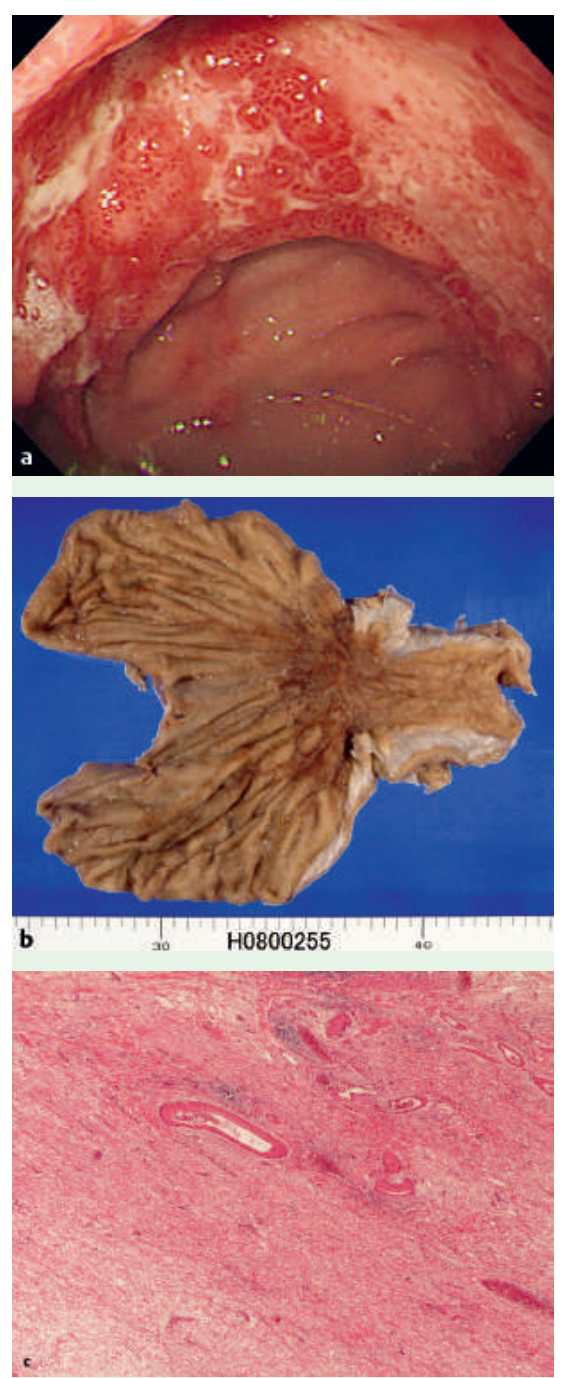

Endoscopy_UCTN_Code_CCL_1AB_2AD_3AD

Endoscopy_UCTN_Code_CCL_1AB_2AD_3AC

\section{T. Terada}

Department of Pathology, Shizuoka Municipal Shimizu Hospital, Shizuoka, Japan

\section{References}

1 Usui Y, Matsukawa M, Hamada Tet al. Corrosive gastritis mimicking linitis plastica carcinoma. Gastroenterol Jpn 1989; 24: 398 401

2 Lagasse JP, Causse X, Legoux JL et al. Cytomegalovirus gastritis simulating cancer of linitis plastica on endoscopic ultrasonography. Endoscopy 1998; 30: S101-S102

Bibliography

DOI 10.1055/s-2008-1077664

Endoscopy 2009; 41: E90

(c) Georg Thieme Verlag KG Stuttgart · New York . ISSN 0013-726X

Corresponding author

\section{T. Terada, MD, PhD}

Shizuoka Municipal Shimizu Hospital

Miyakami 1231

Shimizu-ku

Shizuoka

424-8636

Japan

Fax: +81-54-3341173

piyo0111jp@yahoo.co.jp 